\title{
Urinary excretion of pyridinium crosslinks in healthy $4-10$ year olds
}

Shahid M Husain, Zulf Mughal, Gwen Williams, Kate Ward, Colin S Smith, John Dutton, William D Fraser

\begin{abstract}
Urinary pyridinoline and deoxypyridinoline, pyridinium crosslinks released during breakdown of mature collagen, might serve as useful markers of bone resorption. Before their role can be identified, reference values must be established. In this study, free pyridinoline (f-Pyr), free deoxypyridinoline (f-DPyr), and creatinine $(\mathrm{Cr})$ were measured in first morning void urine samples from 250 girls and 265 boys between the ages of 4 and 10 years. Overall, there was a decrease in f-Pyr: $\mathrm{Cr}$ and $f-D P y r: C r$ ratios with increasing age in both sexes, but there was a wide range of values for individuals of similar ages. Further studies are required to assess whether urinary pyridinium crosslink excretion is sufficiently deranged in conditions affecting bone metabolism for the measurement of these compounds to be of clinical value.

(Arch Dis Child 1999;80:370-373)
\end{abstract}

Department of Child

Health, St

Bartholomew's and the

Royal London School

of Medicine and

Dentistry, Homerton

Hospital, London

E9 6SR, UK

$S$ M Husain

Department of

Paediatric Medicine,

St Mary's Hospital,

Manchester M13 0JH,

UK

Z Mughal

K Ward

Institute of Child

Health, Royal

Liverpool Children's

Hospital, Liverpool

L12 2AP, UK

G Williams

C S Smith

Department of Clinical Chemistry, Faculty of Medicine, University

of Liverpool, Liverpool

L69 3GA, UK

J Dutton

W D Fraser

Correspondence to: Dr S Husain, Department of Neonatology, Homerton Hospital, Homerton Row, London E9 6SR, UK.

Accepted 26 November 1998
Keywords: pyridinoline; deoxypyridinoline; bone resorption

Certain biochemical markers of bone turnover might prove to be of clinical use in conditions and treatments that affect bone metabolism. Markers of bone formation include osteocalcin, bone specific alkaline phosphatase, and propeptides derived from type I collagen, all of which have the disadvantage of requiring a serum sample for their measurement; whereas markers of bone resorption, including calcium, hydroxyproline, hydroxylysine glycosides, telopeptides and pyridinium collagen crosslinks, can be measured in urine. Until recently, urinary hydroxyproline was the best established marker of bone resorption. However, urinary hydroxyproline is influenced by dietary intake, is metabolised extensively in the liver, and is not specific for bone collagen. ${ }^{1}$ Pyridinoline (Pyr) and deoxypyridinoline (DPyr) are non-reducible pyridinium compounds that crosslink mature collagen chains within extracellular matrices and are released into the circulation during collagen resorption. Unlike DPyr is not influenced by dietary intake ${ }^{1}$ and is more specific for resorption of bone collagen. ${ }^{2}$ In adults, $~ 30-40 \%$ of pyridinium crosslinks appear free in urine, with the remainder in peptide form. $^{23}$

The measurement of total urinary pyridinium crosslinks in adults has shown good correlation between Pyr and DPyr excretion and hydroxyproline, urinary excretion of Pyr and bone turnover as assessed by radioisotopic ${ }^{4}$ and histomorphometric ${ }^{5}$ techniques, but corresponding data in children are not available. The clinical usefulness of measuring pyridinium crosslinks in adult urine has been shown in a variety of bone diseases including rheumatoid arthritis, osteoarthritis, primary hyperparathyroidism, hyperthyroidism, Paget's disease, osteomalacia, osteoporosis, and metastatic bone disease. ${ }^{6}$

If the urinary excretion of Pyr and DPyr is to be of any value in assessing bone resorption in conditions or treatments that might affect bone turnover in children, then normal ranges for the excretion of these crosslinking amino acids must be established. However, there are few data on urinary pyridinium crosslink excretion in healthy children. Shaw et al measured urinary total Pyr and DPyr in 109 children between the ages of 2 and 15 years attending hospital for day case surgery and demonstrated a wide variation in their excretion between and within subjects. ${ }^{7}$ However, these children cannot be strictly termed healthy because they were undergoing surgery. The aims of our study were to establish reference ranges for the urinary excretion of free pyridinoline (f-Pyr) and free deoxypyridinoline (f-DPyr) and examine their relation with age in healthy children aged 4-10 years. f-Pyr and f-DPyr were measured using a method developed in Liverpool, UK, which has improved assay turnaround time and assay sensitivity.

\section{Methods}

Our study was approved by the Central Manchester and Royal Liverpool Children's Hospital ethics committees. Head teachers of primary schools in these areas were contacted to obtain permission for the study to be carried out. If granted, a parental information sheet, consent form, instructions for urine collection, and sterile universal containers were supplied to all children. Parents who agreed to allow their children to participate in our study were asked to supply details of any acute or chronic illness affecting their child and were requested to collect a first morning void urine sample from their child on a predetermined date and send the sample and signed consent form with the child to school. The samples were collected

Table 1 Mean (95\% confidence limits) height and weight SDS of study population

\begin{tabular}{lrl}
\hline & Height & Weight \\
\hline Boys & $0.13(-0.003$ to 0.25$)$ & $0.45^{\star}(0.33$ to 0.57$)$ \\
Girls & $-0.03(-0.16$ to 0.10$)$ & $0.27^{\star}(0.15$ to 0.40$)$ \\
\hline
\end{tabular}

${ }^{\star} \mathrm{p}<0.0001$ compared to 0 (Student's $t$ test, two tailed). 


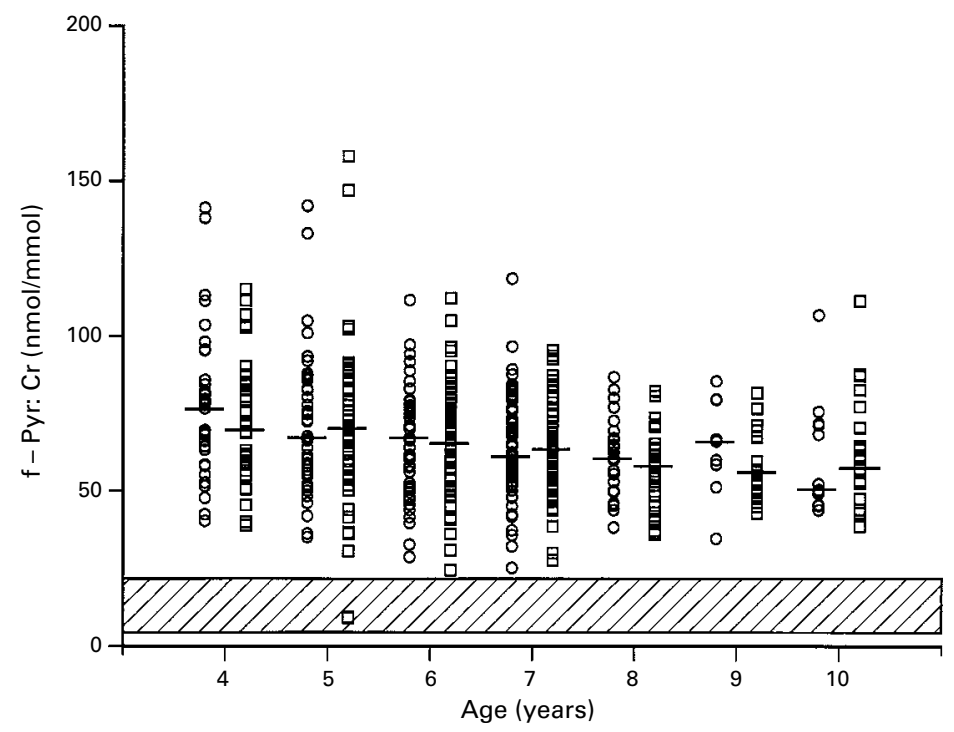

Figure 1 Urinary free pyridinoline:creatinine ratio (f-Pyr:Cr) in 250 girls (circles) and 265 boys (squares) aged 4 to 10 years. Medians are shown as bars and the hatched area represents the adult reference range.

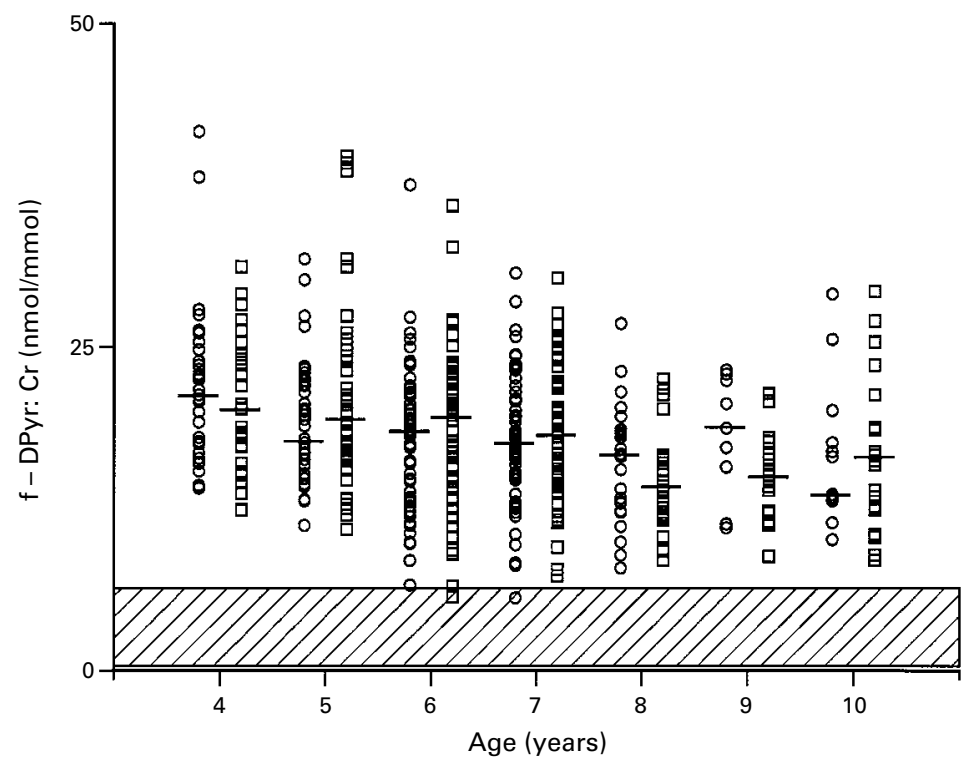

Figure 2 Urinary free deoxypyridinoline:creatinine ratio (f-DPyr:Cr) in 250 girls (circles) and 265 boys (squares) aged 4 to 10 years. Medians are shown as bars and the hatched area represents the adult reference range.

Table 2 Urinary free pyridinoline:creatinine $(f-P y r: C r)$ and free deoxypyridinoline:creatinine ( $f$-Dpyr:Cr) ratios in children aged 4 to 10 years

\begin{tabular}{|c|c|c|c|c|c|}
\hline \multirow[b]{2}{*}{ Age (years) } & \multirow[b]{2}{*}{$n$} & \multicolumn{2}{|c|}{ f-Pyr:Cr (nmol:mmol) } & \multicolumn{2}{|c|}{ f-DPyr:Cr (nmol:mmol) } \\
\hline & & Median & $I Q$ range & Median & $I Q$ range \\
\hline \multicolumn{6}{|l|}{ Boys } \\
\hline 4 & 31 & 69.1 & 58.7 to 85.0 & 20.2 & 17.4 to 24.3 \\
\hline 5 & 47 & 69.9 & 54.7 to 83.0 & 19.4 & 15.9 to 22.5 \\
\hline 6 & 56 & 65.7 & 53.0 to 76.7 & 19.6 & 14.8 to 22.3 \\
\hline 7 & 59 & 63.4 & 51.6 to 72.2 & 18.1 & 14.6 to 22.1 \\
\hline 8 & 28 & 57.8 & 46.1 to 63.4 & 14.1 & 12.0 to 16.1 \\
\hline 9 & 21 & 56.3 & 49.5 to 67.3 & 15.0 & 12.3 to 16.3 \\
\hline 10 & 23 & 57.3 & 45.7 to 67.4 & 16.6 & 12.6 to 18.8 \\
\hline \multicolumn{6}{|l|}{ Girls } \\
\hline 4 & 35 & 76.6 & 63.7 to 85.1 & 21.3 & 17.2 to 25.1 \\
\hline 5 & 41 & 67.6 & 55.7 to 82.9 & 17.6 & 14.8 to 22.1 \\
\hline 6 & 58 & 67.1 & 57.6 to 77.5 & 18.5 & 13.8 to 21.2 \\
\hline 7 & 70 & 61.3 & 55.3 to 75.1 & 17.5 & 15.2 to 21.0 \\
\hline 8 & 26 & 60.6 & 49.9 to 66.9 & 16.7 & 12.6 to 18.4 \\
\hline 9 & 9 & 66.0 & 58.5 to 79.4 & 18.7 & 15.7 to 22.4 \\
\hline 10 & 11 & 50.0 & 45.5 to 71.2 & 13.5 & 13.1 to 17.6 \\
\hline
\end{tabular}

IQ, interquartile. from the school and deep frozen at $-20^{\circ} \mathrm{C}$ within four hours. In addition, the children's weight and height were measured using Seca scales and either a Karrimeter or Minimeter (Raven Equipment, Welwyn Garden City, Herts, UK), and their decimal age calculated using the date of birth in the school register. Measurements were made with the children wearing their school uniform without shoes or socks.

A modification of the high performance liquid chromatography (HPLC) method of Black $^{8}$ was used to measure urinary f-Pyr and f-DPyr, as described previously. ${ }^{9}$ Briefly, acidified urine was applied to microgranular cellulose (CC31) in butanol (1/4) and washed before elution with heptafluorobutyric acid $(0.1 \%)$. The eluent was then analysed by ion pair reverse phase HPLC using fluorescence detection. Acetylated Pyr (Metra Biosystems, Oxford) was used as an internal standard. The interassay coefficient of variation for both methods is $<5.5 \%$ across the working concentration for the assay in our laboratory. To allow for differences in urine concentration, f-Pyr and f-DPyr were expressed as a ratio compared with urinary creatinine (Cr), which was measured by means of standard automated techniques (Boehringer Mannheim, Lewes, Sussex, UK).

Because the effect (if any) of ethnicity on urinary pyridinium crosslink excretion is unclear, ${ }^{10}$ only the data on white children are presented here; ethnicity was self reported by parents. Children suffering from any illness or receiving long term medication (as identified by their parents) were excluded from the analysis. The results were banded into age groups of 4 to $<5$ years $=4$ years; 5 to $<6$ years $=5$ years; and so on. To determine how closely the anthropometric data of the study population resembled that of the general population, the height and weight standard deviation score (SDS) for each child was calculated using the British 1990 growth reference data ${ }^{11}$ and the mean SDS values for the whole study population were compared to zero by means of the Student's $t$ test (two tailed). Changes with age were assessed by linear regression and differences between sexes by comparing the slope and $y$ intercept of the linear regression lines for boys and girls.

\section{Results}

Urinary Cr, f-Pyr, and f-DPyr were measured in first morning void samples from 250 girls and 265 boys between the ages of 4 and 10 years. The mean and $95 \%$ confidence limits of the height and weight SDS values are shown in table 1 . In both sexes the mean weight SDS was significantly greater than zero.

The results of urinary f-Pyr and f-DPyr, expressed as a ratio compared with urinary $\mathrm{Cr}$, in relation to age are shown in figs 1 and 2, respectively, together with the adult reference range in our laboratory (450 subjects (90 men) aged 30-79 years). Almost all the values in children are greater than the values seen in adults but, as can be seen, there is a wide variation within each age group in both sexes. 
There was overall variability in the f-Pyr: $\mathrm{Cr}$ and f-DPyr: $\mathrm{Cr}$ ratios, with a tendency to decrease with increasing age $(p<0.01)$. For either marker, there were no significant differences between the slopes and y intercepts of the linear regression lines in boys compared with girls. Therefore, the data were combined and the linear regression equations are $\mathrm{y}=-3.2(\mathrm{x})+88.2$ and $\mathrm{y}=-1.0(\mathrm{x})+25.4$ for f-Pyr:Cr and f-DPyr:Cr, respectively; where y is the urinary metabolite to creatinine ratio and $\mathrm{x}$ is the decimal age of the child. Table 2 shows median and interquartile ranges of f-Pyr and f-DPyr excretion for both sexes at each age group in our study.

There were weak inverse correlations $(r=-0.2$ to -0.3$)$, which were highly significant, between free pyridinium crosslink excretion and height and weight in both sexes (data not shown).

\section{Discussion}

Our study has demonstrated enhanced excretion of f-Pyr and f-DPyr in primary school children compared with our laboratory's reference values for adults. Similar findings for total Pyr and DPyr have been reported in 24 hour urine samples ${ }^{12}{ }^{13}$ and first morning void urine samples, ${ }^{74-16}$ and indicate the high rate of bone turnover in young subjects. The measurement of urinary free pyridinium crosslinks in large numbers of children has not been reported previously, but in adults the proportion of free crosslinks is $\sim 40 \%$ in normal volunteers and patients with a wide range of different diseases, and it appears that the same might be true in healthy children. ${ }^{2}$ There are reports that the proportion of free crosslinks excreted in urine is related to the rate of bone turnover. ${ }^{17} \mathrm{With}$ this in mind, our results are consistent with total Pyr and DPyr normative data reported from healthy children in continental Europe $^{131516}$ and Japan. ${ }^{14}$ In addition, our community based results are consistent with a hospital based study carried out in the UK.?

In agreement with other studies, we found considerable variability in crosslink excretion between subjects of the same age group. This variability might be explained partly by the uncertainty of the timing of the urine sample in our subjects. Although parents were requested to supply first morning void urine samples from their children, it is not possible to know whether they complied with our request. However, between subject and within subject variability is considerable, even when allowance is made for the circadian rhythm. ${ }^{76}$ Shaw et al demonstrated a within subject variability of $10-30 \%$ in a small number of individuals in whom a first morning void sample of urine was collected consecutively over five days ${ }^{7}$; Marowska et al reported similar within subject variability. ${ }^{16}$ Nevertheless, our data show an overall decline in free crosslink excretion between the ages of 4 and 10 years, at least in girls, with perhaps a small increase in values in boys after the age of 8 to 9 years (table 2). These findings are consistent with the notion that the rate of bone turnover, and hence bone resorption, slows during the primary school years, which is reflected in a corresponding decrease in published height velocity rates during this time period. This is supported by the finding in healthy children of a strong association between published growth velocity rates and urinary collagen type I crosslinked $N$-telopeptides (used as a marker of bone resorption) in a cross sectional study, ${ }^{18}$ and measured growth velocity and 24 hour urinary total Pyr and DPyr excretion in a longitudinal study. ${ }^{13}$

Urinary excretion of total DPyr and Pyr (and presumably f-Pyr and f-DPyr) is subject to a circadian rhythm, with higher rates of excretion at night. ${ }^{12}{ }^{14}$ There are inconsistent reports on the correlation between measurements made on 24 hour urine collections and early morning samples, ${ }^{3141920}$ and it is unknown whether pyridinium crosslink excretion measured in a 24 hour urine sample offers any advantage over the use of a first morning void sample. In postmenopausal women, measurement of DPyr and Pyr in first morning void urine samples more closely reflects the rate of bone loss compared with the measurement in 24 hour urine samples. ${ }^{19}$ In addition, 24 hour urine collections are troublesome and difficult to obtain in children who are not hospitalised. Unless quantification of bone resorption is required, it is likely that assessment of changes in bone resorption or derivation of normative data can be made on spot urine samples collected at similar times. For these reasons, we chose to study pyridinium crosslink excretion in first morning void urine samples rather than 24 hour urine samples.

We did not find any sex related differences, whereas Marowska et al found the urinary excretion of total Pyr and DPyr to be significantly greater in girls than boys in first morning void urine samples at the age of 8 years. ${ }^{16}$ This difference might be a result of the onset of puberty and the accompanying growth spurt in girls, but pubertal assessment was not carried out either in our study or that of Marowska.

We found weak inverse correlations, which were highly significant, between urinary free pyridinium crosslink excretion and height and weight in both sexes. Marowska et al reported more moderate inverse correlations $(r=-0.5$ to -0.7 ) in a larger number of subjects. ${ }^{16}$ These weak to moderate correlations might be another indication of the between subject variability discussed above. As can be seen from table 1, the mean weight SDS of our study population was significantly greater than zero for both boys and girls, suggesting that the weights of children in our study were on average greater than the 50th centile of growth charts in current use. Whether this difference is real or represents a cohort effect is unclear.

Previous studies in children, who acted as their own controls, have used urinary excretion of total Pyr and DPyr to examine the effect on bone resorption of inhaled steroids in children with asthma ${ }^{2122}$ and growth hormone treatment in children with growth hormone deficiency. ${ }^{71319}$ However, because of the large between and within subject variation in urinary 
pyridinium crosslink excretion and the overlap between normative data and that obtained from children with conditions that might affect bone turnover, the clinical value of measuring urinary pyridinium crosslink excretion requires further investigation. There is considerable derangement of the DPyr:Pyr ratio in patients with Ehlers Danlos syndrome type VI, largely as a result of increased urinary excretion of DPyr, ${ }^{23}$ but whether the same is true in other conditions of interest is not known. The normative data provided by our study might be helpful in establishing the role of urinary collagen crosslinks in childhood bone disorders.

We thank the pupils and their parents and teachers for participating in our study.

1 Russell RGG. The assessment of bone metabolism in vivo using biochemical approaches. Horm Metab Res 1997;29:138-44.

2 Robins SP. Biochemical markers for assessing skeletal growth. Eur f Clin Nutr 1994;48(suppl 1):S199-209.

3 Abbiati G, Bartucci F, Longoni A, et al. Monitoring of free abbiati G, Bartucci F, Longoni A, et al. Monitoring of free and total urinary pyridinoline and deoxypyridinoline in healthy volunteers: sample relationships between $24 \mathrm{hr}$ and fasting early

4 Eastell R, Colwell A, Hampton L, Reeve, J. Biochemical markers of bone resorption compared with estimates of bone resorption from radiotracer kinetic studies in osteoporosis. $\mathcal{F}$ Bone Miner Res 1997;12:59-65.

5 Delmas PD, Schlemmer A, Ginetys E, Riis B, Christiansen C. Urinary excretion of pyridinoline crosslinks correlates with bone turnover measured on iliac crest biopsy in patients with vertebral osteoporosis. 7 Bone Miner Res 1991;6:639-44.

6 Pyridinium crosslinks as markers of bone resorption [editorial]. Lancet 1992;340:278-9.

7 Shaw NJ, Dutton J, Fraser WD, Smith CS. Urinary pyridinoline and deoxypyridinoline excretion in children. Clin Endocrinol 1995;42:607-12.

8 Black D, Duncan A, Robins SP. Quantitative analysis of the pyridinium crosslinks of collagen using ion-paired reversed-phase high performance liquid chromatography. Anal Biochem 1988;169:197-203.

9 MacDonald AG, Birkinshaw G, Durham B, Bucknall RC, Fraser WD. Biochemical markers of bone turnover in sero- negative spondylarthropathy: relationship to disease activity. Br f Rheumatol 1997;36:50-3.

10 Pratt JH, Manatunga AK, Peacock M. A comparison of the urinary excretion of bone resorptive products in white and black children. $\mathcal{F}$ Lab Clin Med 1996;127:67-70.

11 Freeman JV, Cole TJ, Chinn S, Jones PR, White EM, Preece MA. Cross-sectional stature and weight reference curves for the UK, 1990. Arch Dis Child 1995; 73:17-24.

12 Eastell R, Simmons PS, Colwell A, et al. Nyctohemeral changes in bone turnover assessed by serum bone Gla-protein concentration and urinary deoxypyridinoline Gla-protein concentration and urinary deoxypyridinoline excretion:

13 Rauch F, Schönau E, Woitge H, Remer T, Seibel M. Urinary excretion of hydroxy-pyridinium cross-links of collagen reflects skeletal growth in normal children. Exp Clin Endocrinol 1994;102:94-7.

14 Fujimoto S, Kubo T, Tanaka H, Miura M, Seino Y. Urinary pyridinoline and deoxypyridinoline in healthy children and in children with growth hormone deficiency. $f$ Clin Endocrinol Metab 1995;80:1922-8.

15 Açil Y, Brinckmann J, Notbohm H, Müller PK. Changes with age in the urinary excretion of hydroxylysylpyridinoline (HP) and lysylpyridinoline (LP). Scand $\mathcal{F}$ Clin Lab Invest 1996;56:275-83.

16 Marowska J, Kobylińska M, ukaszkiewicz J, Taajko A, Rymkiewicz-Kluczyńska B, Lorenc RS. Pyridinium crosslinks of collagen as a marker of bone resorption rates in children and adolescents: normal values and clinical application. Bone 1996;19:669-77.

17 Fraser WD. The collagen crosslinks pyridinoline and deoxypyridinoline: a review of their biochemistry, measurement, and clinical applications. Fournal of Clinical Ligand Assay 1998;21:102-10.

18 Bollen AM, Eyre, DR. Bone resorption rates in children monitored by the urinary assay of collagen type I cross-linked peptides. Bone 1994;15:31-4.

19 Uebelhart D, Schlemmer A, Johansen JS, Ginetys E, Christiansen C, Delmas PD. Effect of menopause and hormone replacement therapy on the urinary excretion of pyridin-
ium crosslinks. F Clin Endocrinol Metab 1991;72:367-73.

20 McLaren AM, Isdale AH, Whiting PH, Bird HA, Robins SP. Physiological variations in the urinary excretion of pyridinium crosslinks of collagen. Br F R heumatol 1993;32:307-12.

21 Birkebæk NH, Esberg G, Andersen K, Wolthers O, Hassager C. Bone and collagen turnover during treatment with inhaled dry powder budesonide and beclomethasone dipropionate. Arch Dis Child 1995;73:524-7.

22 Doull I, Freezer N, Holgate S. Osteocalcin, growth, and inhaled steroids: a prospective study. Arch Dis Child 1996;74:497-501.

23 Pasquali M, Dembure PP, Still MJ, Elsas LJ. Urinary pyridinium cross-links: a noninvasive diagnostic test for EhlersDanlos syndrome type VI. N Engl f Med 1994;331:132-3. 